\title{
Thin-Film Microtensile-Test Structures for High-Throughput Characterization of Mechanical Properties
}

\author{
T. Oellers, V. G. Arigela, C. Kirchlechner, G. Dehm,* and A. Ludwig*
}

Cite This: ACS Comb. Sci. 2020, 22, 142-149

ABSTRACT: A photolithographic process for the rapid fabrication of thin-film tensile-test structures is presented. The process is applicable to various physical vapor deposition techniques and can be used for the combinatorial fabrication of thin-film tensile-test structure materials libraries for the high-throughput characterization of mechanical properties. The functionality of the fabrication process and the feasibility of performing high-quality measurements with these structures are demonstrated with $\mathrm{Cu}$ tensile-test structures. In addition, the scalability from unary structures to libraries with compositional variations is demonstrated.

KEYWORDS: thin film, micromechanical testing, combinatorial materials science, physical vapor deposition, high-throughput experimentation

\section{INTRODUCTION}

Significant progress in the field of micromachining technology has enabled many electromechanical applications of miniaturized material systems. But with decreasing dimensions, microelectromechanical and microelectronic systems face the challenge of increasing thermomechanical load during operation. This can lead to an increased failure rate due to, e.g., electromigration induced mechanical failure or stress induced delamination. ${ }^{1-3}$ To reduce failure rate and improve device performance simultaneously, it is important to develop new thin-film materials with improved and tailored functional properties. Over time, combinatorial methods have shown their high capabilities in accelerating material development and optimization. The core of combinatorial materials science is the utilization of high-throughput processes to synthesize and characterize a high number of samples in the form of a library. Such a library is superimposed with a well-controlled property gradient (e.g., chemical composition, microstructure, structure size) which is fabricated during synthesis or during postprocessing. Subsequently, the functional properties of interest are characterized along the gradient to identify optimal material performance. A significant challenge for combinatorial approaches is the availability of characterization techniques which can provide necessary throughput, as many techniques are limited by measurement time, or sample preparation.

To improve lifetime of microsystems, the knowledge of thinfilm mechanical properties is important, as many failures are interconnected with mechanical behavior. An additional challenge for the application of materials in microsystems is that materials do show size-dependent mechanical behavior with typically increasing strength at smaller dimensions. ${ }^{4-10}$ Therefore, it is required to improve knowledge about properties on a length scale which is representative of the system where the material is applied. In addition to scaling effects that result from the length scale of structures on a device, there are many additional influences on the mechanical behavior which depend on the microstructure (e.g., grain size, intrinsic stress, crystal orientation, phase formation) or the utilized test setup (e.g., resolution, force application, alignment inaccuracies). ${ }^{11-13}$ For this work, emphasis is placed on the general concept of combinatorial test-structure fabrication on a predefined length-scale. Influences on the mechanical properties that are caused by microstructural changes are expected and intended as a result of the compositional gradient that is fabricated during combinatorial synthesis. Within the combinatorial approach, microstructural changes are one of the main parameters that are used to influence functional properties. Further influences of the test setup, especially sample alignment, are not considered in depth here, as these issues primarily depend on the capabilities of the setup itself. On the sample side, approaches such as self-aligning tensile structures $^{14}$ can help to alleviate misalignments, but this requires complex, three-dimensional structuring which is difficult to integrate into a high-throughput approach.

Received: November 1, 2019

Revised: February 18, 2020

Published: February 18, 2020 


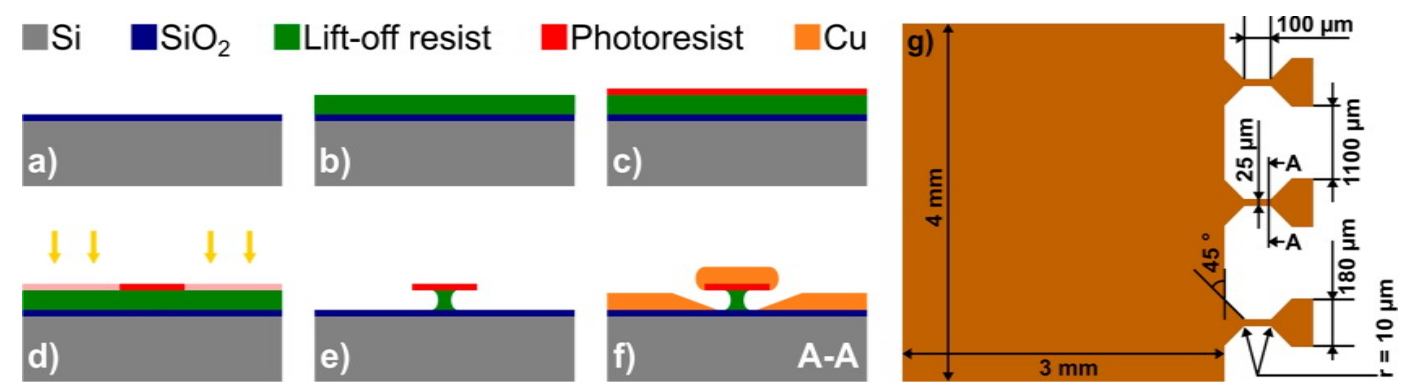

Figure 1. Schematic process flow for the fabrication of tensile-test structures (cross sections, not to scale). (a) Starting with a thermally oxidized Si wafer, followed by (b, c) spin-coating of a bilayer lift-off resist system. The resist system is structured by (d) photolithography and (e) development. In the last step, the tensile-test structure material is deposited by magnetron sputtering (f). After deposition, the structures can be released from the resist by immersion in acetone. (g) Schematic dimensions of the fabricated tensile-test structures and handle (not to scale). Structure thickness ranges between 4 and $5 \mu \mathrm{m}$ approximately. Indicated is also the schematic position of the cut A-A (f)).

Investigations of mechanical properties for microscale thin films are typically performed by wafer curvature, ${ }^{15}$ by nanoindentation ${ }^{16}$ on continuous films, or with defined sample geometries by micropillar compression, ${ }^{17,18}$ bending cantilever, ${ }^{7,19}$ or tensile experiments. ${ }^{20-22}$ From these, pillar, cantilever and tensile structure specimens are typically fabricated by focused ion beam (FIB) milling and are well suited to study mechanical properties and scaling effects, because structures can be fabricated with high precision and within a large nano/microscopic range due to the high degree of control during milling. The main limitations for FIB-based combinatorial approaches are connected to the risk of beam damage to the processed material due to implantation of $\mathrm{Ga}$ ions during milling and the slow process speed. Beam damage limits the range of materials that can be processed, while process speed limits the number of samples that can be effectively fabricated for individual studies. This makes the integration of FIB milling into combinatorial structure fabrication difficult, as the latter requires fabrication processes that can be universally applied for varying materials with defined compositional gradients and high-throughput capability.

Examples which demonstrate the fabrication of thin-film structures for micromechanical testing on a wafer-scale can be found in the literature. ${ }^{23-28}$ But these usually require dedicated and expensive micromachining equipment such as reactive ion etching, ion milling, or chemical vapor deposition of dielectrics such as $\mathrm{SiO}_{2} / \mathrm{Si}_{3} \mathrm{~N}_{4}$. In this study, we present an alternative approach for the fabrication of thin-film tensile-test structures based on a photolithographic lift-off process. Photolithographic processes, which are well established throughout the microsystems/-electronics industry, are well suited for combinatorial approaches as they enable the fabrication of a high quantity of structures on the scale of, typically, Si wafers. Structures can be fabricated with well-defined and repeatable geometries. In labscale processes, laser lithography and mask aligner systems are typically utilized for rapid photolithographic processes which allow structure fabrication with a resolution from hundreds of nanometers up to the dimension of the used substrate. But as wafer-scale photolithography is typically used to transfer twodimensional patterns, the main geometrical limitation during fabrication is the achievable structure thickness which is constrained by the thickness of photoresists and deposition techniques. With such an approach, it is possible to achieve repeatable structure fabrication in both a rapid and parallel process which allows for high-throughput mechanical characterization in a tensile-test configuration.

\section{EXPERIMENTAL SECTION}

Tensile-Test Structure Fabrication Process and Material Synthesis. Thin-film tensile-test structures were fabricated in a photolithographic process which was designed to enable rapid fabrication of tensile-test structure arrays on a 4 in. diameter wafer scale and for compatibility with combinatorial codeposition.

Thermally oxidized 4 in. diameter Si (100) wafer substrates with a $1.5 \mu \mathrm{m}$ thick $\mathrm{SiO}_{2}$ diffusion barrier were used as a substrate for the fabrication process (Figure 1a). In a first step, a bilayer of non-photosensitive lift-off photoresist (LOR $20 \mathrm{~B}$, MicroChem Corp.) with a combined thickness of approximately $6.6 \mu \mathrm{m}$ was spin-coated (Figure 1b). The resist thickness was selected to be higher than the nominal thickness of the tensile-test structures (in the order of $2 / 3$ of the resist thickness) to ensure a successful lift-off process. The two layers were deposited by two successive spin coating steps which were performed with a waiting time of $5 \mathrm{~s}$ in between the coatings. The individual coatings were performed with a dynamic dispense at a rotational speed of approximately 8.31 / $\mathrm{s}(500 \mathrm{rpm})$ over a time of $5 \mathrm{~s}$, with a final rotation speed of approximately $16.7 \mathrm{1} / \mathrm{s}(1000 \mathrm{rpm})$ for $35 \mathrm{~s}$ and an acceleration of approximately $183.3 \mathrm{rad} / \mathrm{s}^{2}(1750 \mathrm{rpm} / \mathrm{s})$. After deposition of the bilayer, a soft bake was performed at $160{ }^{\circ} \mathrm{C}$ for $120 \mathrm{~s}$. In the next step, a positive tone photoresist with a thickness of approximately $1.5 \mu \mathrm{m}$ (AZ1518, Microchemicals) was spin-coated (Figure 1c). The dispense was performed dynamically at a rotational speed of approximately $8.31 / \mathrm{s}(500 \mathrm{rpm})$ over a time of $5 \mathrm{~s}$, with a final rotation speed of approximately $66.7 \mathrm{1} / \mathrm{s}(4000 \mathrm{rpm})$ for $30 \mathrm{~s}$ and an acceleration of approximately $183.3 \mathrm{rad} / \mathrm{s}^{2}(1750 \mathrm{rpm} / \mathrm{s})$. The soft bake was performed at $100{ }^{\circ} \mathrm{C}$ for $50 \mathrm{~s}$. Exposure of the top resist was performed with a laser lithography system ( $\mu \mathrm{PG}$ 101, Heidelberg Instruments Mikrotechnik $\mathrm{GmbH}$ ) with a dose of approximately $55 \mathrm{~mJ} / \mathrm{cm}^{2}$ (Figure $1 \mathrm{~d}$ ). After exposure, the resist system was processed by immersion development in an aqueous-alkaline developer (AZ 726 MIF, Microchemicals) for approximately $60 \mathrm{~s}$, followed by a rinsing cascade in deionized water (Figure 1e).

After fabrication of the resist lift-off structure, the deposition of the tensile-test structure material was performed (Figure 1f). For deposition, a magnetron sputter system with a confocal target setup was used. The thin film was grown by 
simultaneous sputtering of two diametrically opposed $\mathrm{Cu}$ targets (4 in. diameter, 99.999 atom \% purity). The deposition powers (direct current) for both $\mathrm{Cu}$ targets were adjusted for a similar sputter rate of approximately $0.18-0.2 \mathrm{~nm} / \mathrm{s}$. For the fabrication of $\mathrm{Cu}-\mathrm{Ag}$ thin-film libraries, depositions were performed with an additional $\mathrm{Ag}$ target (4 in. diameter, 99.99 at. \% purity), positioned orthogonally to the $\mathrm{Cu}$ targets. Depositions were performed with an additional substrate bias ( $3 \mathrm{~W}, \sim 52 \mathrm{~V}$, radio frequency) at a pressure of approximately $0.133 \mathrm{~Pa}(1 \mathrm{mT}$ Trr) with an Ar plasma at room temperature and a base pressure $<2.7 \times 10^{-5} \mathrm{~Pa}$. Deposition time was adjusted to achieve a film thickness of up to $5 \mu \mathrm{m}$. Sputter parameters were adjusted to achieve low stress variations and low porosity within the freestanding films. Low sputter pressure and additional bias were used to achieve increased incident energy during deposition, resulting in dense films. Literature sources were used as an initial guide to achieve stress compensation: low sputter pressure, typically resulting in compressive stress, could be compensated by substrate bias, which can result in tensile stress. ${ }^{29-31}$

After deposition of the tensile-test structure material, the liftoff was performed by dissolving the top photoresist layer in acetone. The bottom resist layer was selected to be insoluble in acetone, ensuring a clean and consistent lift-off of the tensile structure. Lift-off was continued until the structures were fully detached from the substrate and photoresist residues on the structure surface were fully dissolved. After lift-off, the structures were removed from the acetone individually and cleaned with a rinsing cascade of acetone and isopropanol to remove possible contaminations.

Layout of the Tensile-Test Structures. The overall design of a fabricated sample encompasses two main elements. The tensile-test structure itself for mechanical characterization and a rectangular section, which is utilized as a handle for manual manipulation and clamping in the tensile-test apparatus. The in-plane geometry of the structures is defined by the photoresist lift-off structure.

The nominal dimensions of the tensile-test structures are shown in Figure 1g) The main dimensions important for mechanical testing are gauge length, gauge width, and film thickness (nominal values: 100,25 , and $5 \mu \mathrm{m}$, respectively). Corners in the transition area from the gauge to the "head" and handle structures were designed with a radius of $10 \mu \mathrm{m}$ to reduce stress concentrations. For wafer-scale fabrication, structures were arranged in a square array ( $4.5 \mu \mathrm{m}$ pitch) with 15 rows and 14 columns for a total of 210 structures with three tensile-test structures each. Dimensions were selected to follow "DIN EN ISO 6892-1" for structures with rectangular cross sections as closely as possible, following the approach by Smolka et al. ${ }^{27}$. Structure thickness and gauge length had to be adjusted in comparison to the geometry used by Smolka et al. to a nominal thickness of $5 \mu \mathrm{m}$ and a gauge length of $100 \mu \mathrm{m}$. Thickness was adjusted for compatibility with the selected liftoff process and to limit deposition time to ensure that the deposition can be performed with the utilized sputter system in a single and continuous process. The gauge length was reduced to reflect the reduced cross-sectional area of the gauge. To follow the norm as closely as possible, a length of $100 \mu \mathrm{m}$ was selected, as a smaller value was not possible due to the physical size of the utilized gripper which required this minimum length for insertion of the structure. The handle section was designed as big as possible within the confines of the measurement area of $4.5 \mathrm{~mm}$ by $4.5 \mathrm{~mm}$. With a handle area of $4 \mathrm{~mm}$ by $3 \mathrm{~mm}$, the structure can be manually gripped and positioned with tweezers without damaging the tensile-test structures.

Tensile-Test Experiments. Tensile-test experiments were performed with an UNAT 2 indenter (ASMEC GmbH). The setup can be equipped with different gripping and indenter devices to allow for universal mechanical testing. For this work, a Si gripper (see Smolka et al. ${ }^{27}$ ), fabricated by deep reactive ion etching with the inverse shape of the "head" of the tensiletest structure, was used for applying load to the tensile-test structures. Tensile tests were performed in displacementcontrolled mode with displacement rates of 400 and $40 \mathrm{~nm} / \mathrm{s}$. The setup was placed in a scanning electron microscope (SEM; JEOL JSM-6490) for testing.

For testing, the thin-film structures are attached to a holder which is then fitted into the test setup. In a first step, the structures are fixed in between two rectangular $\mathrm{Si}$ plates with Ag glue (G 3692, PLANO GmbH), resulting in a mechanically more stable assembly for subsequent handling and attachment to the holder. In a second step, the plate/structure assembly is attached to a universal structure holder with Ag glue (Figure 2 ). The holder and tensile setup are connected by a circular rod at the setup and a matching hole at the base of the holder which is fixed in place with a set screw.

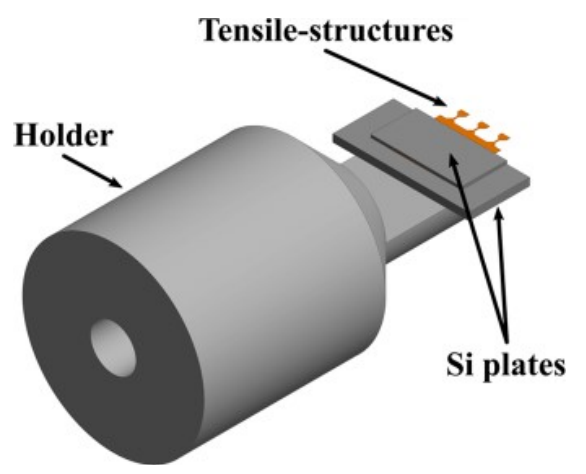

Figure 2. Schematic assembly of the tensile-test structures and the holder that is fitted into the test setup. The structures are fixed in between two Si plates which are then attached to the holder.

For alignment, the actuators of the setup allow free positioning in the $x / y / z$ direction. Rotational alignment around the long axis of the gauge is possible by rotation of the holder around the circular rod that is used for attachment. Other rotational axes are aligned during attachment of the tensile-test structures to the holder and cannot be changed afterward in this setup. During prepositioning for testing, the structures were placed as close to the bottom surface of the gripper as possible. The procedure was to approach the gripper surface with a low speed until a first contact in between both structures was recognized. Subsequently, the tensile-test structure is moved away again slightly into the position where direct contact is lost, which is the final position before testing.

Characterization of the Tensile-Test Structures. The geometry of the tensile-test structures was analyzed by electron microscopy images of the tensile-test structure surface and cross sections through the gauge length. Cross sections were prepared by low energy ion milling with $\mathrm{Ar}$ ions (IM4000Plus Ion milling system, Hitachi) and electron imaging was performed with a SEM (JEOL 7200F). 
Thickness profiles of as-deposited films were obtained by using a tactile profilometer (Ambios XP 2) with a scan speed of $0.1 \mathrm{~mm} / \mathrm{s}$ and a stylus load of $20 \mathrm{mg}$. Substrate-bound films that remained on the $\mathrm{Si}$ wafer after lift-off were utilized to measure thickness profiles.

The composition of the as-deposited $\mathrm{Cu}-\mathrm{Ag}$ materials library was obtained by energy dispersive X-ray spectroscopy (EDS) using a JEOL JSM-5800LV SEM with an Oxford instruments INCA $\mathrm{x}$-act EDS detector. EDS measurements were performed with an acceleration voltage of $20 \mathrm{kV}$, working distance of $10 \mathrm{~mm}$, and $600 \times$ magnification. Compositional data was obtained with a measurement accuracy of \pm 1 atom $\%$.

Microstructure was investigated by electron backscatter detector (EBSD) analysis (Oxford Instruments Symmetry) in a SEM (JEOL JSM-7200F). Cross-sectional samples for analysis were prepared by low energy Ar-ion milling with Ar ions (IM4000Plus Ion milling system, Hitachi).

\section{RESULTS AND DISCUSSION}

Analysis of the Structure Geometry. For evaluation of the fabrication process and mechanical testing, $\mathrm{Cu}$ tensile-test structures were fabricated. A freestanding tensile-test structure is shown in Figure 3a. With the selected process parameters,
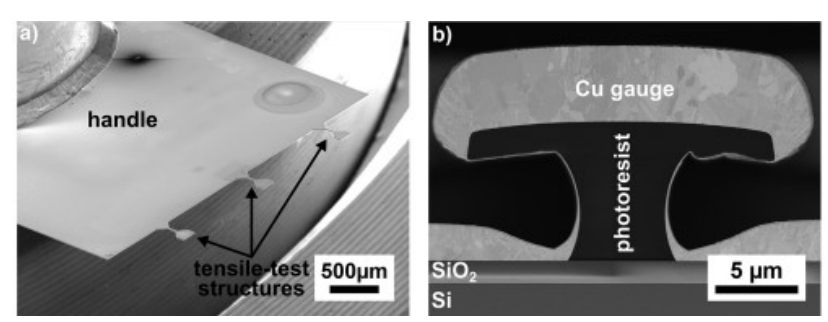

Figure 3. (a) SEM image of a fabricated thin-film structure with a handle and three tensile-test structures. Circular discoloration in the corner of the handle structure is caused by residues remaining during evaporation of isopropanol during the last cleaning step. (b) SEM image of a cross section through the gauge of a $\mathrm{Cu}$ tensile-test structure, orthogonal to the gauge before release of the structure from the bilayer photoresist.

the structures remain in a flat shape after lift-off, which is of high importance for the mechanical testing. To characterize the actual structure dimensions and influences of the polymer structure on the final geometry, cross sections of the tensiletest structures were prepared before lift-off while the structures are still attached to the Si substrate. The structure shown in Figure $3 \mathrm{~b}$ ) has a width and thickness of approximately 23 and $4.6 \mu \mathrm{m}$, respectively. Deviations of the structure thickness from the nominal value of $5 \mu \mathrm{m}$ were caused by variations over the tensile structure array due to the sputter configuration with increasing thickness for substrate positions closer to the sputter sources. The top photoresist structure shows a curvature along the width of the gauge section which is reproduced in the deposited $\mathrm{Cu}$ film (Figure $3 \mathrm{~b}$ )). The curvature is attributed to insufficient mechanical stability of the top resist layer due to the undercut of the lift-off resist underneath. During the development step of the fabrication process a lateral undercut with a size similar to the thickness of the lift-off resist is generated. The undercut is required for the lift-off step but approximately two-thirds of the top resist remain mechanically unsupported, resulting in the deformation. An additional influence on the geometry is deposition on the sidewalls of the top resist layer, resulting in a triangular shaped overhang at the edge of the gauge. The deposition on the sidewall also extends to the side of the gauge area, resulting in a semicircular sidewall geometry with increasing film thickness. The geometry at the sides is mainly caused by the confocal setup of the deposition system. With the low sputter pressure, the material flux is generally inclined toward a line of sight characteristic which allows for more pronounced deposition on the structure sidewalls.

Both, the curvature and the overhang result in a processrelated deviation from a rectangular cross-section. The crosssectional area of the gauge was measured to be approximately $105 \mu \mathrm{m}^{2}$ by image analysis, which takes all geometrical features such as curvature along the gauge width and the semicircular deposition on the sides into account. Coincidentally, this area is close to the area of a rectangle without geometrical deviations with a cross-sectional area of $105.8 \mu \mathrm{m}^{2}$ for the same dimensions of $23 \mu \mathrm{m}$ width and $4.6 \mu \mathrm{m}$ height. A drawback of the triangular shaped overhang is the possibility of stress concentrations in that area during loading which could result in premature failure. This effect could be amplified when load imperfections (e.g., misalignment) are present, which might be detrimental in particular for brittle systems.

Execution of the tensile-test experiments required a specific orientation of the structure in the tensile-test apparatus. Initial tests showed significant deformations at the head of the tensiletest structures which resulted in undefined mechanical loading which cannot be used to probe the material properties (Figure 4a). It was observed that, under load, the head structure started

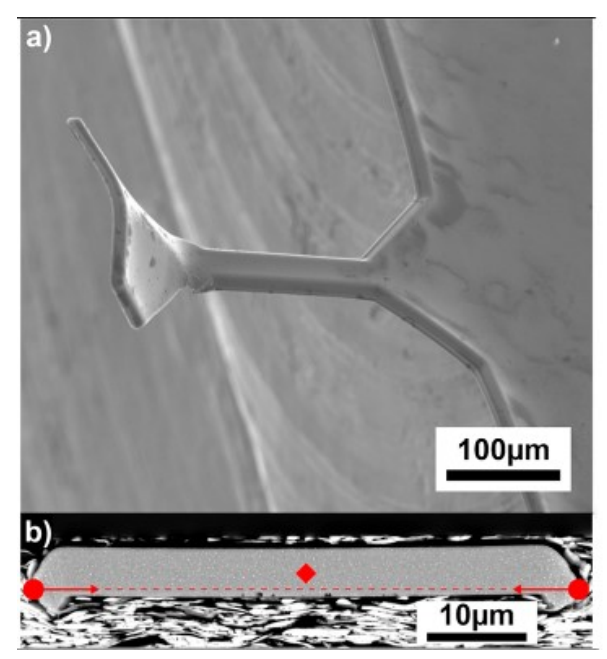

Figure 4. (a) SEM image of a failed tensile-test experiment due to bending and buckling of the structure head. The head is bent in the direction of the top surface. The experiment was stopped before fracture to illustrate the deformation. (b) Cross-sectional cut through the head of a $\mathrm{Cu}-\mathrm{Ag}$ structure. Indicated are the position of the first contact of the gripper structure with the tensile-test structure $(\mathbf{})$, the direction of the applied force $(\rightarrow)$, and the approximate center of mass $(\checkmark)$ of the cross section.

buckling and bent in direction of the top surface. The deformation was caused by the shape of the structure sidewalls (semicircular) and gripper (straight). Upon loading, the initial contact in between the gripper and tensile-test structure occurs at the outer edges of the rounded sidewalls. In an ideal case, the force is applied in horizontal direction as illustrated by red arrows in Figure $4 \mathrm{~b}$. The direction of the applied force is offset 
from the center of mass of the tensile gauge. Due to this effect, a momentum is induced, which results in an initial upward bending of the head structure followed by subsequent buckling around the long axis of the structure. To counteract buckling of the head, the tensile-test structures had to be placed with the bottom surface facing upward in the gripper structure. Additionally, the test structure was positioned in contact with the bottom surface of the gripper before the tensile-test. In this configuration, the moment was still induced upon loading but buckling of the structure head was constrained by the bottom surface of the gripper. To prevent prebending, the gripper and structure were not placed in direct contact, but with a remaining gap $(\leq 1 \mu \mathrm{m})$ before testing. Due to the gap, an out-of-plane bending of the structure head toward the gripper will still occur during the experiment which can cause unwanted strain at the transition from the gauge to the head. With the distance in between both structures, a bending of approximately $<0.5^{\circ}$ is expected. This behavior makes it critical that fracture does not occur close to the structure head to perform valid experiments.

An additional artifact from the fabrication process was observed at the bottom surface of the structure. Localized at the edges of the structure, an additional, thin layer can be seen (Figure 5). The layer forms during thin film deposition where a
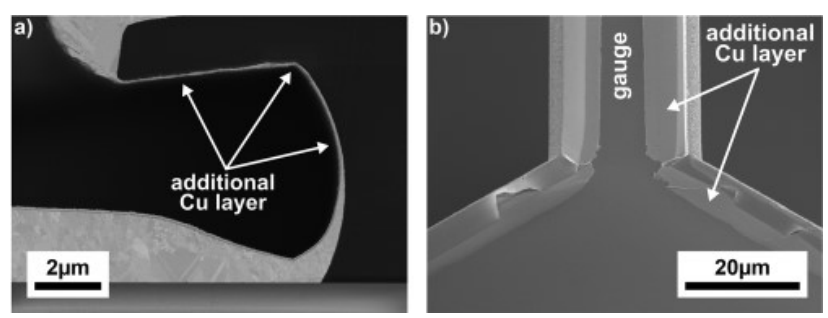

Figure 5. SEM images: (a) Magnified view of a cross-section cut through the gauge on the lift-off photoresist. A thin $\mathrm{Cu}$ layer is located along the bottom and sidewall of the photoresist surface. (b) Tensiletest structure bottom surface at the transition region from the head to the gauge. An attached layer with a thickness of $<20 \mathrm{~nm}$ is observed toward the edge of the tensile structure.

small fraction of sputtered atoms coats the underside of the photoresist structure by scattering in the Ar plasma (Figure 5a). During lift-off, the layer fractures at the connection in between the top and bottom resist layers, which is a mechanical weak point. After fracture, the layer on the underside of the top resist attaches to the bottom surface of the structure and remains after drying (Figure $5 \mathrm{~b}$ ). The layer has a thickness of $<20 \mathrm{~nm}$, approximately $0.4 \%$ of the structure thickness. During execution of the tensile-tests, no detrimental effects on the stress-displacement data which correlate with failures in the artifact layer were observed. This was attributed to the low thickness relative to the structure thickness. But the layer can disturb image analysis during and after testing.

Tensile-Test Experiments. For validation of the fabrication method and functionality of the tensile-test structures, pure $\mathrm{Cu}$ structures were fabricated and tensile-tests were performed in the as-deposited state. The resulting stressdisplacement graphs of two different, exemplary tensile experiments are shown in Figures $6 \mathrm{a}$ and 7 . The two experiments were selected to illustrate different effects that can occur during testing and their influence on the measurement.
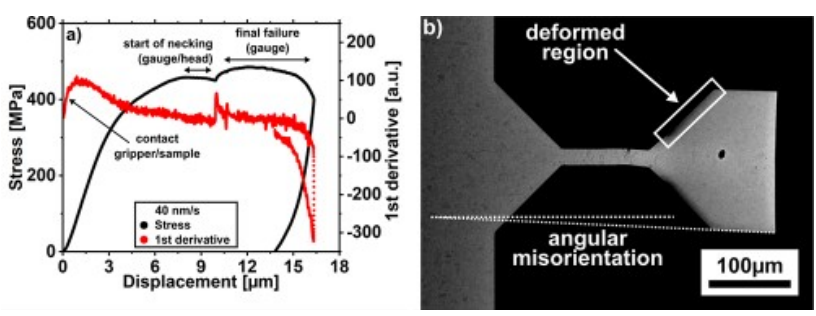

Figure 6. (a) Graphs showing the stress-displacement data of a tensile-test experiment and the corresponding first derivative (red). The experiment was performed with a displacement rate of $40 \mathrm{~nm} / \mathrm{s}$. The experiment was stopped before fracture for postmortem image acquisition. (b) Backscatter electron SEM image of a strained tensiletest structure before fracture.

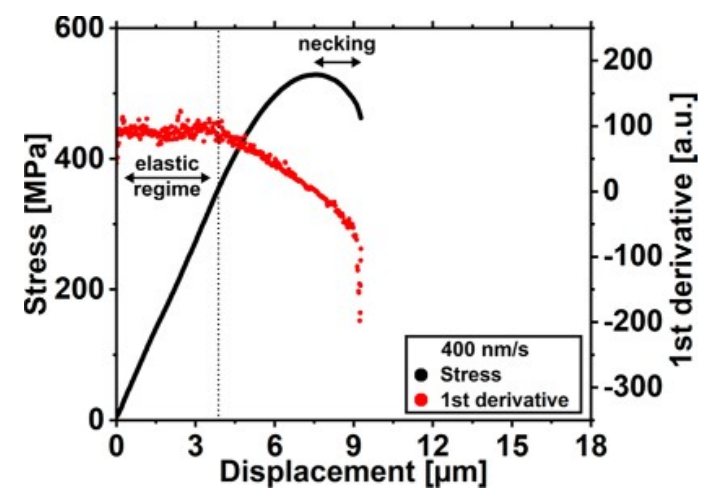

Figure 7. Stress-displacement data of a tensile-test experiment and the corresponding first derivative (red). The experiment was performed with a displacement rate of $400 \mathrm{~nm} / \mathrm{s}$ and the tensile-test structure was strained until fracture.

The first experiment (Figure 6a) was performed with a displacement rate of $40 \mathrm{~nm} / \mathrm{s}$ (strain rate of $\sim 4 \times 10^{-4} \mathrm{~s}^{-1}$ ), and the test was stopped before fracture to allow for postmortem analysis of the full tensile structure. In an ideal case, after initial contact of the gripper and the sample head, the stress should increase linearly with increasing displacement in the elastic regime. For this experiment, the stressdisplacement does not show a linear regime and the slope increases directly after first contact of the gripper and structure. This is observed more clearly by plotting the first derivative (Figure 6a) of the stress-displacement data, as it directly represents the slope. The initial increase for the first derivative is a result of a nonsymmetric contact with the gripper and subsequent rotational deformation of the sample head as indicated in Figure $6 \mathrm{~b}$. This deformation is superimposed with the elastic regime, and therefore, it is not possible to measure the Young's modulus of the tested material. The initial misalignment might also be responsible for the overall shape of the curve with the two distinct stress maxima. The maxima correlate with necking in the gauge and the formation of an additional crack at the transition region from the gauge to the sample head. The formation of the necking and the crack correlate with the first stress plateau. Propagation of the crack stops at the end of the first stress plateau. Necking in the gauge continues with the failure correlating with the second stress plateau. The crack at the sample head might be caused by the deformation induced by the initial angular misalignment in between the structure and gripper. The alignment error was estimated by postmortem image analysis to be approximately 
$2.5^{\circ}$ (Figure 6b). Additionally, the image analysis shows deformations of the structure head in the contact area with the gripper (Figure $6 \mathrm{~b}$ ). The deformation in the displacement direction was estimated to be approximately $1.5-2 \mu \mathrm{m}$. It is assumed that the deformation was caused by the small contact area of the vertical gripper and rounded structure sidewall during the initial contact (Figure 4b). With increasing deformation, the contact area increases, which results in a decrease of the local stress until no further deformation occurs. Both the displacement from the plastic deformation within the gauge section and additional deformation effects from the clamping are summed up in the stress-displacement data. As a result, it is not possible to confidently identify the elastic regime and transition from elastic to plastic deformation with the associated mechanical properties. In this case, the ultimate tensile strength is the main parameter that can be utilized for characterization, as it reflects the load that was definitively carried by the gauge and therefore the minimum strength of the material.

A second experiment (Figure 7) was performed with a displacement rate of $400 \mathrm{~nm} / \mathrm{s}$ (strain rate of $\sim 4 \times 10^{-3} \mathrm{~s}^{-1}$ ) until fracture. The setup was well aligned, and no significant deformation of the tensile-test structure upon initial contact with the gripper was observed, as indicated by the linear behavior of the stress-displacement data. This is supported by the first derivative curve (Figure 7), showing an approximately constant slope for the elastic regime. With the better alignment only a single instance of necking occurs, followed by fracture in the gauge. The reduction of alignment-related errors allows for evaluation of the linear elastic part of the stress-displacement curve. For this experiment, the Young's modulus is evaluated as approximately $90 \mathrm{GPa}$, which is approximately $23 \%$ lower compared to that in the literature. The compliance of the system was not considered for the experiments. Digital image correlation, as used by Smolka et al. ${ }^{27}$ in a comparable tensiletest setup, could be utilized here as well to obtain information regarding system compliance which would help to provide more realistic data on the elastic material behavior and accurate strain values. Due to these uncertainties, it is difficult to accurately separate elastic and plastic regimes and subsequently to evaluate Young's moduli. Therefore, the ultimate tensile strength (UTS) is used as the main parameter for mechanical characterization in this approach. Both experiments show similar behavior during failure and approximate UTS of 485 and $528 \mathrm{MPa}$, respectively. The average grain size of as-deposited $\mathrm{Cu}$ tensile-test structures was approximately $360 \mathrm{~nm}$ in the gauge area. The UTS values are in good agreement with literature values for tensile-test experiments which feature microscale dimensions or nanoscale grain sizes, if grain size dependent effects on the mechanical properties (e.g., Hall-Petch) are considered. ${ }^{27,32-39}$

Application for High-Throughput Experimentation. Beyond testing of tensile-test structures consisting of a single element, the fabrication process was specifically developed for combinatorial approaches, utilizing physical vapor deposition for library fabrication. To demonstrate the applicability for this purpose, a tensile-test structure library with a binary $\mathrm{Cu}-\mathrm{Ag}$ composition gradient was fabricated.

Compositional and thickness variation as well as two exemplary photographs of the library are shown in Figure 8. For this exemplary library, the Ag content ranged from 4 to 9 atom \% (Figure 8a) with structure thicknesses between 4.2 and $5 \mu \mathrm{m}$ (Figure $8 \mathrm{~b}$ ). A section of the materials library containing
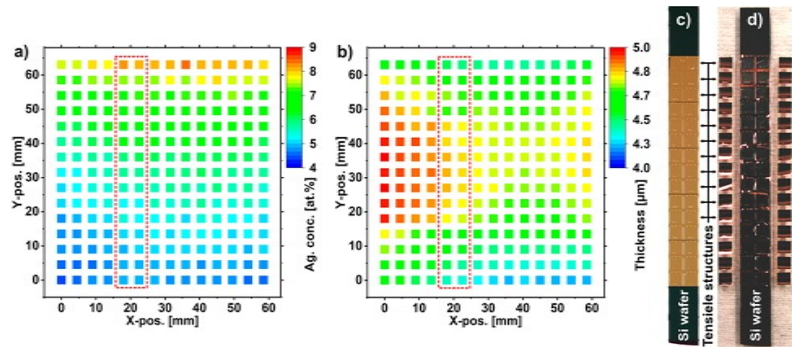

Figure 8. Plots of the (a) composition and (b) thickness of a binary $\mathrm{Cu}-\mathrm{Ag}$ tensile structure materials library. The measurement areas correspond the fabricated tensile-test structures. $Y$ - and $X$-positions correspond to the lateral positions of the measurement areas on the substrate. (c) Tensile-test structures on a substrate after deposition and before lift-off. The stripe is cleaved from a Si wafer containing the full materials library. (d) Tensile-test structures removed from the substrate. For visualization, the structures are placed beside the corresponding position on the substrate after detachment. The scale is consistent throughout all images.

two sets of 15 measurement areas with tensile-test structures is shown in Figure $8 \mathrm{c}$ and d. The position of the cleaved stripe on the full material library is marked in Figure $8 \mathrm{a}$ and $\mathrm{b}$ by the red, dashed outline. Figure $8 \mathrm{c}$ shows tensile-test structures after deposition on the photoresist and before lift-off from the $\mathrm{Si}$ substrate (see Figure 1f). The stripe was cleaved from the wafer substrate with the full materials library to simplify the removal of the tensile-test structure by reducing the quantity of measurement structures that need to be handled in one batch. The removal of the tensile-test structures is illustrated in Figure $8 \mathrm{~d}$. Here, the substrate is immersed in acetone to dissolve the photoresist and disconnect the tensile-test structures from the substrate. For illustration, the thin-film structures are placed beside the substrate stripe in correspondence with the original $Y$-position. Due to the image scale, only the rectangular handle section of the structures is clearly visible. The compositional and thickness ranges are defined by the geometry of the confocal sputter setup. To cover a bigger compositional range, multiple materials libraries have to be fabricated, or alternative sputtering techniques such as "wedge-type" multilayer deposition with moveable shutters must be used. ${ }^{40}$

Due to the use of multiple sputter sources, we observe the controlled compositional gradient along the $Y$-axis (Figure 8a) and an additional variation in film thickness (Figure $8 \mathrm{~b}$ ) which was quantified for the individual measurement areas displayed in the graphs. As the gradients also exist within the measurement area $(4.5 \mathrm{~mm} \times 4.5 \mathrm{~mm})$, we also have thickness and compositional variation over the three tensile-test structures within an individual measurement area. For the depicted materials library, the length axis of the tensile structure gauge was positioned along the compositional gradient ( $Y$-axis in Figure $8 \mathrm{a}$ ) and the three tensile-test structures belonging to one measurement area are positioned along the $X$-axis (Figure $8 \mathrm{~b}$ ). With these orientations, the average thickness variation in between two neighboring structures within a measurement area can be calculated to be approximately $7 \mathrm{~nm}$ which corresponds to approximately $0.15 \%$ of the average thickness. The average compositional variation along $100 \mu \mathrm{m}$ of the gauge length was calculated to be 0.005 atom \%. Both variations are small when compared to the absolute values of the respective measurement areas, and 
the influence on the obtained mechanical properties is expected to be insignificant.

With the test setup used, approximately two tensile-tests can be performed per hour. As the setup is intended for universal use, the main limitation concerning throughput is manual handling for attachment to sample holders, fitting holders into the setup, and alignment, which accounts for more than $50 \%$ of the machine time. The main aspects to increase throughput are an improved sample fixture and automation of structure positioning and alignment. Improved fixture mechanisms can be designed to prealign and directly clamp the tensile-test structures at the handle without preprocessing. For the exemplary testing of the structures, the use of a universal setup was sufficient, but for efficient high-throughput testing of an entire materials library an optimized testing setup is required.

\section{CONCLUSIONS}

A high-throughput approach for tensile testing of thin-film structures was presented. The process was adapted to be applicable to PVD deposition of materials with either homogeneous compositions or compositional gradients.

Structure fabrication and testing was demonstrated with elementary $\mathrm{Cu}$ test structures. Structure analysis revealed geometrical deviations which had to be considered during testing to perform a repeatable and representative investigation of mechanical behavior. The ultimate tensile strength was selected as the main mechanical parameter, which could be characterized with high confidence. The investigation of further parameters was difficult due to interactions between the tensile-test structures and the gripping device during displacement. Future work could be executed to further improve the fabrication process to reduce geometrical deviations and subsequently improve accuracy and repeatability of the tensile-tests.

The process was then transferred to wafer-scale fabrication of tensile-test structure materials libraries with a compositional variation, which is essential for the utilization in a combinatorial approach. This was exemplarily demonstrated by deposition of a binary $\mathrm{Cu}-\mathrm{Ag}$ alloy materials library. Analysis showed that tensile-test structures with a defined compositional variation were fabricated which could be used for combinatorial testing.

With the photolithographic high-throughput process, it was possible to fabricate more than 600 tensile-test structures within a single day of processing which presents a significant increase in fabrication speed in comparison to typical FIB based processes. The utilized SEM indenter setup allowed for a throughput of approximately two tensile-tests per hour which represented the main limitation for testing. As such, additional work is required to improve automation and to design a dedicated tensile setup to simplify structure handling, clamping, and alignment to further improve measurement throughput.

\section{AUTHOR INFORMATION}

\section{Corresponding Authors}

G. Dehm - Max-Planck-Institut für Eisenforschung $\mathrm{GmbH}$, 40237 Düsseldorf, Germany; Email: dehm@mpie.de

A. Ludwig - Institute for Materials, Ruhr-Universität Bochum, 44801 Bochum, Germany; 이이.org/0000-0003-2802-

6774; Email: alfred.ludwig@rub.de

\section{Authors}

T. Oellers - Institute for Materials, Ruhr-Universität Bochum, 44801 Bochum, Germany

V. G. Arigela - Max-Planck-Institut für Eisenforschung GmbH, 40237 Düsseldorf, Germany

C. Kirchlechner - Max-Planck-Institut für Eisenforschung GmbH, 40237 Düsseldorf, Germany; ○ orcid.org/0000-00032418-9664

Complete contact information is available at:

https://pubs.acs.org/10.1021/acscombsci.9b00182

\section{Notes}

The authors declare no competing financial interest.

\section{ACKNOWLEDGMENTS}

T.O. and V.G.A. acknowledge a $\mathrm{PhD}$ fellowship from the International Max Planck Research School for Surface and Interface Engineering (IMPRS-SurMat). The use of scientific infrastructure of the $\mathrm{ZGH}$ at RUB is acknowledged. G.D. acknowledges financial support by the European Research Council (ERC) under the EU's Horizon 2020 Research and Innovation Program (Grant No. 787446-GB-CORRELATE).

\section{REFERENCES}

(1) $\mathrm{Tu}, \mathrm{K} . \mathrm{N}$. Recent advances on electromigration in very-largescale-integration of interconnects. J. Appl. Phys. 2003, 94, 5451-5473.

(2) Lloyd, J. R. Electromigration and mechanical stress. Microelectron. Eng. 1999, 49, 51-64.

(3) Arzt, E.; Kraft, O.; Spolenak, R.; Joo, Y.-C. Physical metallurgy of electromigration: Failure mechanisms in miniaturized conductor lines. Z. Metallkd./Mater. Res. Adv. Tech. 1996, 87, 934-942.

(4) Hall, E. O. The Deformation and Ageing of Mild Steel: III Discussion of Results. Proc. Phys. Soc., London, Sect. B 1951, 64, 747753.

(5) Petch, N. J. The Cleavage Strength of Polycrystals. J. Iron Steel Inst. 1953, 174, 25-28.

(6) Armstrong, R. W. The influence of polycrystal grain size on several mechanical properties of materials. Metall. Mater. Trans. B 1970, 1, 1169-1176.

(7) Nix, W. D. Mechanical properties of thin films. Metall. Trans. A 1989, 20, 2217.

(8) Dehm, G. Miniaturized single-crystalline fcc metals deformed in tension: New insights in size-dependent plasticity. Prog. Mater. Sci. 2009, 54, 664-688.

(9) Kraft, O.; Gruber, P. A.; Mönig, R.; Weygand, D. Plasticity in Confined Dimensions. Annu. Rev. Mater. Res. 2010, 40, 293-317.

(10) Greer, J. R.; De Hosson, J. Th. M. Plasticity in small-sized metallic systems: Intrinsic versus extrinsic size effect. Prog. Mater. Sci. 2011, 56, 654-724.

(11) Read, D. T.; Dally, J. W. A new method for measuring the strength and ductility of thin films. J. Mater. Res. 1993, 8, 1542-1549.

(12) Hazra, S. S; Baker, M. S; Beuth, J. L; de Boer, M. P Demonstration of an in situ on-chip tensile tester. J. Micromech. Microeng. 2009, 19, 082001.

(13) Hazra, S. S.; Baker, M. S.; Beuth, J. L.; de Boer, M. P. Compact On-Chip Microtensile Tester With Prehensile Grip Mechanism. J. Microelectromech. Syst. 2011, 20, 1043-1053.

(14) Kang, W.; Saif, M. T. A. A Novel Method for In Situ Uniaxial Tests at the Micro/Nano Scale-Part I: Theory. J. Microelectromech. Syst. 2010, 19, 1309-1321.

(15) Doerner, M. F.; Gardner, D. S.; Nix, W. D. Plastic properties of thin films on substrates as measured by submicron indentation hardness and substrate curvature techniques. J. Mater. Res. 1986, 1, 845-851. 
(16) Pethicai, J. B.; Hutchings, R; Oliver, W. C. Hardness measurement at penetration depths as small as $20 \mathrm{~nm}$. Philos. Mag. A 1983, 48, 593-606.

(17) Uchic, M. D.; Dimiduk, D. M.; Florando, J. N.; Nix, W. D. Sample Dimensions Influence Strength and Crystal Plasticity. Science 2004, 305, 986-989.

(18) Dehm, G.; Wörgötter, H. P.; Cazottes, S.; Purswani, J. M.; Gall, D.; Mitterer, C.; Kiener, D. Can micro-compression testing provide stress-strain data for thin films?: A comparative study using $\mathrm{Cu}, \mathrm{VN}$, TiN and W coatings. Thin Solid Films 2009, 518, 1517-1521.

(19) Hong, S.; Weihs, T. P.; Bravman, J. C.; Nix, W. D.; Barnett, D. M.; Smith, D. A. Residual stresses in thin films using microcantilever beams In Thin Films: Stresses and Mechanical Properties. Mater. Res. Soc. Proc. 1989, 93-98.

(20) Kiener, D.; Grosinger, W.; Dehm, G.; Pippan, R. A further step towards an understanding of size-dependent crystal plasticity: In situ tension experiments of miniaturized single-crystal copper samples. Acta Mater. 2008, 56, 580-592.

(21) Gianola, D. S.; Eberl, C. Micro- and nanoscale tensile testing of materials. JOM 2009, 61, 24.

(22) Casari, D.; Pethö, L.; Schürch, P.; Maeder, X.; Philippe, L.; Michler, J.; Zysset, P.; Schwiedrzik, J. A self-aligning microtensile setup: Application to single-crystal GaAs microscale tensioncompression asymmetry. J. Mater. Res. 2019, 34, 2517-2534.

(23) Gaspar, J.; Schmidt, M. E.; Held, J.; Paul, O. Wafer-Scale Microtensile Testing of Thin Films. J. Microelectromech. Syst. 2009, 18, 1062-1076.

(24) Bergers, L. I. J. C.; Hoefnagels, J. P. M.; Geers, M. G. D. Onwafer time-dependent high reproducibility nano-force tensile testing. J. Phys. D: Appl. Phys. 2014, 47, 495306.

(25) Magagnosc, D. J.; Ligda, J. P.; Sano, T.; Schuster, B. E. Femtosecond Laser Machining of Micro-tensile Specimens for High Throughput Mechanical Testing. In Micro and Nanomechanics; Starman, L., Hay, J., Eds.; Springer International Publishing: Cham, 2018; Vol. 5, pp 7-9.

(26) Andre, N.; Coulombier, M.; De Longueville, V.; Fabregue, D.; Gets, T.; Gravier, S.; Pardoen, T.; Raskin, J.-P. Microfabrication-based nanomechanical laboratory for testing the ductility of submicron aluminium films. Microelectron. Eng. 2007, 84, 2714-2718.

(27) Smolka, M.; Motz, C.; Detzel, T.; Robl, W.; Griesser, T.; Wimmer, A.; Dehm, G. Novel temperature dependent tensile test of freestanding copper thin film structures. Rev. Sci. Instrum. 2012, 83, 064702.

(28) Pardoen, T.; Colla, M.-S.; Idrissi, H.; Amin-Ahmadi, B.; Wang, B.; Schryvers, D.; Bhaskar, U. K.; Raskin, J.-P. A versatile lab-on-chip test platform to characterize elementary deformation mechanisms and electromechanical couplings in nanoscopic objects. C. R. Phys. 2016, 17, 485-495.

(29) Windischmann, H. Intrinsic stress in sputter-deposited thin films. Crit. Rev. Solid State Mater. Sci. 1992, 17, 547-596.

(30) Lim, J.-W.; Ishikawa, Y.; Miyake, K.; Yamashita, M.; Isshiki, M. Influence of Substrate Bias Voltage on the Properties of $\mathrm{Cu}$ Thin Films by Sputter Type Ion Beam Deposition. Mater. Trans. 2002, 43, 1403-1408.

(31) Pletea, M.; Brückner, W.; Wendrock, H.; Kaltofen, R. Stress evolution during and after sputter deposition of $\mathrm{Cu}$ thin films onto $\mathrm{Si}$ (100) substrates under various sputtering pressures. J. Appl. Phys. 2005, 97, 054908.

(32) Wimmer, A.; Smolka, M.; Heinz, W.; Detzel, T.; Robl, W.; Motz, C.; Eyert, V.; Wimmer, E.; Jahnel, F.; Treichler, R.; et al. Temperature dependent transition of intragranular plastic to intergranular brittle failure in electrodeposited $\mathrm{Cu}$ micro-tensile samples. Mater. Sci. Eng., A 2014, 618, 398-405.

(33) Wimmer, A.; Heinz, W.; Leitner, A.; Detzel, T.; Robl, W.; Kirchlechner, C.; Dehm, G. Micro-tension study of miniaturized Cu lines at variable temperatures. Acta Mater. 2015, 92, 243-254.

(34) Lin, M.-T.; Tong, C.-J.; Shiu, K.-S. Novel Microtensile Method for Monotonic and Cyclic Testing of Freestanding Copper Thin Films. Exp. Mech. 2010, 50, 55-64.
(35) Sanders, P. G.; Eastman, J. A.; Weertman, J. R. Elastic and tensile behavior of nanocrystalline copper and palladium. Acta Mater. 1997, 45, 4019-4025.

(36) Cheng, S.; Ma, E.; Wang, Y. M.; Kecskes, L. J.; Youssef, K. M.; Koch, C. C.; Trociewitz, U. P.; Han, K. Tensile properties of in situ consolidated nanocrystalline Cu. Acta Mater. 2005, 53, 1521-1533.

(37) Wang, Y. M.; Wang, K.; Pan, D.; Lu, K.; Hemker, K. J.; Ma, E. Microsample tensile testing of nanocrystalline copper. Scr. Mater. 2003, 48, 1581-1586.

(38) Zhang, H.; Jiang, Z.; Lian, J.; Jiang, Q. Bulk Nanostructured Cu with High Strength and Good Ductility. Adv. Eng. Mater. 2008, 10, $41-45$.

(39) Zhang, H. Z.; Zhang, H.; Liu, L. Microstructures and Tensile Properties of Electrodeposited $\mathrm{Cu}$ Sheets with Grain Sizes from Nanocrystalline to Ultrafine Scale. In Materials Processing Technology II; Trans Tech Publications, 2012; pp 1611-1614.

(40) Ludwig, A.; Zarnetta, R.; Hamann, S.; Savan, A.; Thienhaus, S. Development of multifunctional thin films using high-throughput experimentation methods. Int. J. Mater. Res. 2008, 99, 1144-1149. 